\title{
Impact of Image-Derived Input Function and Fit Time Intervals on Patlak Quantification of Myocardial Glucose Uptake in Mice
}

\author{
James T. Thackeray, Jens P. Bankstahl, and Frank M. Bengel \\ Department of Nuclear Medicine, Hannover Medical School, Hannover, Germany
}

Limited blood volume in mice precludes repeated sampling, rendering a reliable image-derived input function (IDIF) desirable for quantification of glucose uptake. We aimed to compare different IDIF volumes and to evaluate the effects of changing fit time interval on Patlak uptake kinetics in hearts of healthy mice. Methods: C57BL/6 mice $(n=27)$ were studied under a range of metabolic conditions: no intervention (ctl), overnight fasting, insulin and glucose $(6 \mathrm{mU} / \mathrm{g}, 1 \mathrm{mg} / \mathrm{g})$ under isoflurane, and under ketamine-xylazine anesthesia to suppress glucose uptake. Dynamic PET imaging with ${ }^{18} \mathrm{~F}-\mathrm{FDG}(7.7 \pm 0.9 \mathrm{MBq})$ was conducted. Images were analyzed using left ventricle cavity, left atrial cavity, or inferior vena cava as the IDIF. Patlak analysis was conducted using variable fit time intervals: automated fit, fit from 10 to $60 \mathrm{~min}$ (late), fit from 2 to $30 \mathrm{~min}$ (early), or fit from 2 to 10 min (very early). Results: Both the ventricle and the atrial cavities displayed spill-in from the myocardium in late frames as compared with the vena cava (percentage injected dose per gram, ctl: $21.4 \pm 6.1$ vs. $10.0 \pm 3.9$ vs. $2.5 \pm 0.3, P<0.001$ ). Higher and more rapid passage of peak activity was observed in the vena cava, but the area under the curve over 2 min was similar. The Patlak slope was significantly higher for the vena cava than atrial IDIF (mL/g/min, ctl: $0.11 \pm 0.02$ vs. $0.07 \pm 0.01$; fasting: $0.09 \pm 0.03$ vs. $0.06 \pm 0.02$; insulin: $0.52 \pm 0.09$ vs. $0.23 \pm$ 0.12 ; ketamine-xylazine: $0.001 \pm 0.001$ vs. $0.002 \pm 0.002 ; P<0.01$ ). The rate of glucose uptake was similarly elevated depending on the IDIF $(P<0.01)$. The various IDIF Patlak values were significantly correlated $(r=0.867, P<0.001)$. Automated fit performed reliably in untreated, fasted, and ketamine-xylazine-treated mice, with no statistical difference compared with late, early, or very early fits. The Patlak composite rate constant $\left(\mathrm{K}_{\mathrm{i}}\right)$ was markedly underestimated with automated and late fit after acute insulin treatment, reflecting the rapid early ${ }^{18} \mathrm{~F}-\mathrm{FDG}$ uptake. Conclusion: The choice of IDIF has a profound effect on Patlak kinetics and calculated ${ }^{18} \mathrm{~F}-\mathrm{FDG}$ uptake. Adjustment of the time interval for fit may be necessary for accurate calculations, particularly with acute insulin treatment. Even without partial-volume correction, the inferior vena cava provides a reliable and reproducible IDIF for Patlak analysis of myocardial glucose uptake in mice.

Received May 14, 2015; revision accepted Jul. 31, 2015.

For correspondence or reprints contact: Frank M. Bengel, Department of Nuclear Medicine, Hannover Medical School, Carl Neuberg Strasse 1, D-30625 Hannover, Germany.

E-mail: bengel.frank@mh-hannover.de

Published online Aug. 13, 2015.

COPYRIGHT (C) 2015 by the Society of Nuclear Medicine and Molecular Imaging, Inc.
Key Words: positron emission tomography; fluoro-deoxyglucose; quantitative image analysis; myocardial metabolism; small animal imaging

J Nucl Med 2015; 56:1615-1621

DOI: 10.2967/jnumed.115.160820

$\mathbf{R}$ ecent years have seen a widespread expansion of dedicated small-animal PET systems. This deployment has resulted in a myriad of studies using ${ }^{18} \mathrm{~F}-\mathrm{FDG}$ that apply widely divergent methods for quantification of myocardial glucose utilization $(1,2)$.

The Patlak kinetic model (3) calculates the metabolic rate of glucose utilization based on the composite rate constant, $\mathrm{K}_{\mathrm{i}}\left(K_{1} \times\right.$ $\left.k_{3} /\left(k_{2}+k_{3}\right)\right)$. Patlak graphical analysis expresses tissue uptake relative to dynamic plasma activity, necessitating the acquisition of an accurate plasma input function. The gold standard for determination of input function is repeated arterial blood sampling. Such an approach in mice is complicated by its invasive nature and impractical because of the limited blood volume ( $\sim 2 \mathrm{~mL})$. Consequently, it is inadvisable for multiple-scan longitudinal studies. As such, identification of a reliable image-derived input function (IDIF) is desirable.

In large mammals, the left ventricular cavity can be used to provide a robust time-activity curve for the blood pool. This image-derived volume is less effective in small rodents because limited spatial resolution and partial-volume effects result in significant spill-over from the myocardial tissue in late frames, rendering a falsely high input function that does not converge to zero (4-6). Hybrid approaches have been proposed, defining the peak uptake from early frames using the left ventricle and scaling the input function by a late blood sample $(5,7)$, but the continued dependence on arterial blood sampling complicates serial application. Alternatively, the use of electrocardiogram-gated reconstructions can limit the spill-in to the left ventricular cavity-derived blood pool in late frames $(8,9)$. But the reconstruction of multiple frames into multiple gates is time- and data-intensive, rendering this approach suboptimal for routine application.

In addition to input function complications, the myocardial uptake curve can be widely divergent from the clinical model, with the linear component occurring quite early after tracer injection, manifesting as a relative plateau of tracer uptake in late frames, especially in animals displaying rapid initial uptake. As such, it is necessary to consider adjusting the time intervals for fitting to the Patlak model to optimize the fit to the linear portion of the uptake curve. 
Here, we have applied Patlak graphical analysis to calculate myocardial glucose uptake in C57BL/6 mice under a range of metabolic conditions. With the goal of facilitating a robust, reproducible approach for quantitative analysis in mice, we evaluated the influence of various blood-pool IDIF and fit time intervals on the calculation of Patlak slope and rate of myocardial glucose utilization in comparison with semiquantitative measurements of ${ }^{18} \mathrm{~F}$-FDG accumulation.

\section{MATERIALS AND METHODS}

Further details on methods are provided in the supplemental material (available at http://jnm.snmjournals.org).

\section{Animals}

All experiments were conducted in compliance with European Communities Council Directive 86/609/EEC and 2010/63/EU and were approved by the local animal care committee. C57BL/6 mice ( $n=27$; Charles River Laboratories) were divided to 4 groups of variable glucose uptake: no intervention $(n=10)$; extended fasting $(n=5)$; pretreatment with insulin $(6 \mathrm{mU} / \mathrm{kg})$ and glucose $(1 \mathrm{~g} / \mathrm{kg})$ intraperitoneally $30 \mathrm{~min}$ before ${ }^{18} \mathrm{~F}-\mathrm{FDG}(n=5)$, under standard isoflurane anesthesia; or ketamine $(84 \mathrm{mg} / \mathrm{kg}$, intraperitoneally) and xylazine $(11.8 \mathrm{mg} / \mathrm{kg}$, intraperitoneally) anesthesia $(n=7)$ to suppress myocardial glucose uptake.

\section{PET Imaging}

${ }^{18}$ F-FDG $(7.7 \pm 0.9 \mathrm{MBq})$ was injected via the tail vein, and dynamic 60-min images were acquired in list-mode using a dedicated small-animal PET camera (Inveon DPET; Siemens) as described previously (10).

\section{Image Reconstruction}

List-mode images were histogrammed to 32 frames $(5 \times 2,4 \times 5,3 \times$ $10,8 \times 30,5 \times 60,4 \times 300$, and $3 \times 600 \mathrm{~s})$ and reconstructed using 2 ordered-subset expectation maximization 3-dimensional iterations and 18 maximum a posteriori (OSEM3D/fastMAP) iterations ( $\beta=1$, constant resolution prior) with a target resolution of $1.2 \mathrm{~mm}$.

\section{Image Analysis and Kinetic Modeling}

Images were analyzed using PMOD 3.6 software (PMOD Technologies). Volumes of interest (VOIs) were defined for the left ventricular myocardium $\left(111 \pm 36 \mathrm{~mm}^{3}\right)$ and 3 blood-pool regions-left ventricular cavity $\left(4.2 \mathrm{~mm}^{3}\right.$ sphere), left atrial cavity $\left(4.2 \mathrm{~mm}^{3}\right.$ sphere $)$, or inferior vena cava $\left(12.6 \mathrm{~mm}^{3}\right.$ cylinder between the renal branches and diaphragm) (Supplemental Fig. 1). Myocardium time-activity curves were fitted to a Patlak kinetic model (3) using each blood-pool VOI as IDIF. Temporal fit parameters were varied as follows: automated fit without exclusion; fit from $10 \mathrm{~min}$ (late fit), excluding the early rapid uptake component of the time-activity curve; or fit from 0 to $10 \mathrm{~min}$ (early fit), excluding the plateau component of the time-activity curve. The automated fit considers all 32 points of the curve at equal weighting, generating a linear line of best fit from the earliest aligned time point and excluding points outside of $20 \%$ variance to this fit, irrespective of timing. The rate of myocardial glucose uptake (rMGU) was calculated as $\mathrm{K}_{\mathrm{i}} \times(\mathrm{BG} / \mathrm{LC})$, where $\mathrm{K}_{\mathrm{i}}$ is the graphically defined Patlak slope, $\mathrm{BG}$ is the average blood glucose concentration at the start and end of the scan, and LC is the lumped constant equaling 0.67 as estimated for rodents (4). Patlak values were compared with sequential semiquantitative myocardial ${ }^{18} \mathrm{~F}$-FDG uptake (percentage injected dose per gram $[\% \mathrm{ID} / \mathrm{g}])$.

\section{Statistics}

All data are presented as mean \pm SD. Statistical comparisons were completed using SPSS 18.0 software (IBM).

\section{RESULTS}

\section{Blood-Pool VOI-Derived Input Function}

A wide range of myocardial ${ }^{18} \mathrm{~F}-\mathrm{FDG}$ uptake $(2.8-53.8 \% \mathrm{ID} / \mathrm{g})$ values were obtained by varying scanning conditions (Fig. 1A), with incremental increases in later frames for control and fasted mice. By contrast, myocardial ${ }^{18} \mathrm{~F}$-FDG uptake was maximal in insulin-glucose mice at 10-20 min, with little change in later frames. Ketamine-xylazine mice showed a modest decrease in myocardial ${ }^{18}$ F-FDG over time (Fig. 1B). Time-activity curves demonstrated rapidly rising myocardial activity in control, insulinglucose, and fasted mice under isoflurane anesthesia, whereas myocardial uptake was nearly ablated by ketamine-xylazine anesthesia (Fig. 2). The inferior vena cava exhibited the earliest and highest peak activity $\left(6.27 \pm 2.50 \mathrm{MBq} / \mathrm{cm}^{3}\right)$, significantly higher than in the left atrium $\left(4.61 \pm 2.01 \mathrm{MBq} / \mathrm{cm}^{3}, P<0.05\right)$ and left ventricle $\left(4.02 \pm 1.12 \mathrm{MBq} / \mathrm{cm}^{3}, P<0.01\right)$. The vena cava IDIF declined toward zero over the full course of the scan, whereas the left ventricle and, to a lesser extent, left atrial cavity IDIFs were influenced by spillover from the myocardium. The integral of each IDIF was comparable over the first $120 \mathrm{~s}$, with a higher area under the curve at $5 \mathrm{~min}$ for the left ventricular cavity of insulin-glucose mice. The integral over 5-60 min was consistently higher for the

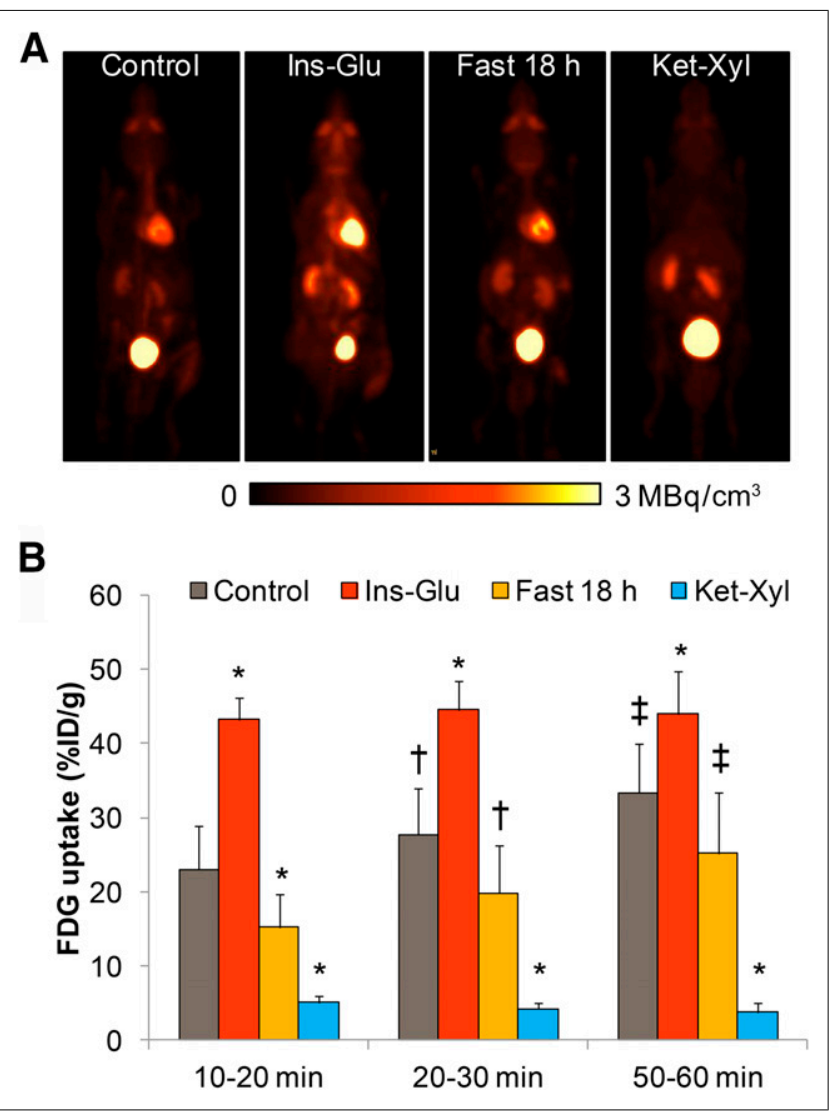

FIGURE 1. Myocardial ${ }^{18} \mathrm{~F}-\mathrm{FDG}$ uptake under various conditions. (A) Representative 3-dimensional maximum-intensity-projection ${ }^{18} \mathrm{~F}-\mathrm{FDG}$ images at 50-60 min after tracer injection. (B) Temporal semiquantitative myocardial ${ }^{18} \mathrm{~F}-\mathrm{FDG}$ uptake as $\% \mathrm{ID} / \mathrm{g}$ in control, insulin-glucose (Ins-Glu) treatment, extended fasting, and ketamine-xylazine (ket-xyl) suppression mice. ${ }^{*} P<0.01$ to control, matched time, 1-way ANOVA. t $P<0.01$ to 10 - to 20 -min frame, matched group, Student paired $t$ test. $\ddagger P<0.01$ to 20 - to 30 -min frame, matched group, Student paired $t$ test. 


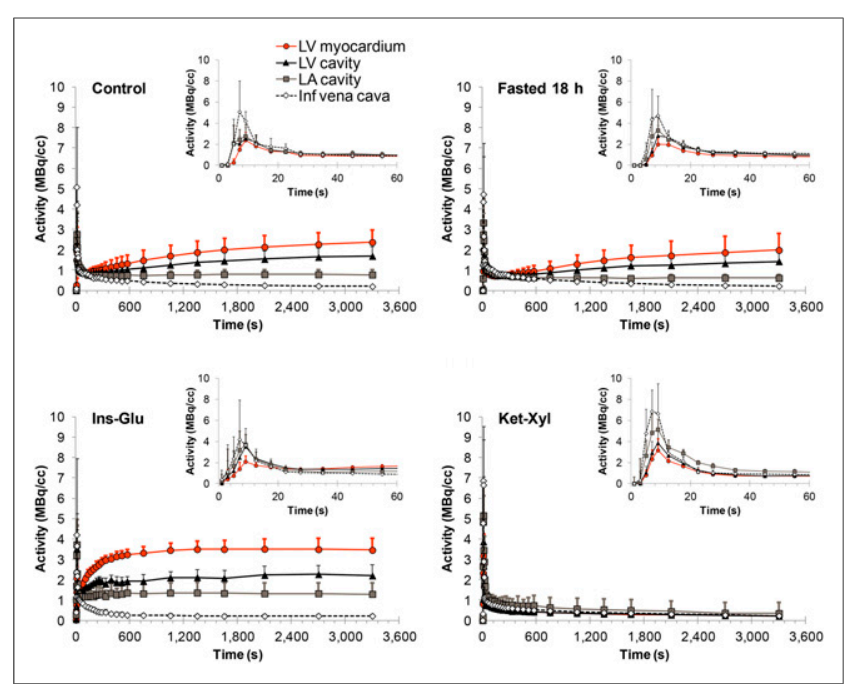

FIGURE 2. Summed time-activity curves. Full time course and first 60 -s (inset) curves display myocardium and 3 blood-pool volumes for IDIF. Ins-Glu = insulin-glucose; Ket-Xyl = ketamine-xylazine.

left ventricular cavity than the left atrium and inferior vena cava, except in the ketamine-xylazine group (Fig. 3).

\section{Impact of IDIF VOI on Patlak Measurements}

Patlak slope $\left(\mathrm{K}_{\mathrm{i}}\right)$ was highly influenced by the selection of blood-pool VOI for IDIF (Supplemental Fig. 2). In control mice, shifting the blood-pool IDIF from the left ventricle to left atrium and to vena cava incrementally increased the automated fit-calculated Patlak $K_{i}$ by 6- and 24-fold, respectively (Fig. 4A). Similar increases were observed in insulin-glucose and fasted mice. There was no difference in ketamine-xylazine Patlak $K_{i}$ calculations. The rMGU was comparably affected in control mice (left atrium, 5.9fold; vena cava, 23.6-fold) and fasted mice (left atrium, 4.5-fold; vena cava, 9.0-fold). The increase was blunted in insulin-glucose mice as reduced blood glucose concentration lowered the rMGU calculation (Fig. 4B; Table 1). Minimal fluctuation was observed in the low-activity ketamine-xylazine group.

\section{Impact of Time Intervals for Fit on Patlak Measurements}

Temporal fit parameters did not severely impact Patlak kinetics using the vena cava IDIF. The automated fit time interval began from $6.3 \pm 4.8 \mathrm{~min}$. In control mice, calculated Patlak slope was comparable for automated, early, and late fits $(r=0.983-0.997$, $P<0.001)$. Similar equivalence was displayed in fasted mice and under ketamine-xylazine suppression (Fig. 5A). Likewise, rMGU was similar within these groups across different fit intervals (Fig. $5 B)$. By contrast, in insulin-glucose mice $K_{i}$ was significantly elevated when the fit was restricted to $2-30 \mathrm{~min}(P=0.01)$, reflecting the rapid initial uptake of ${ }^{18} \mathrm{~F}-\mathrm{FDG}$. Late fit was comparable to automated fit (start from $7.0 \pm 3.4 \mathrm{~min}$ ), because of the plateau of activity after $10 \mathrm{~min}$ of uptake. Differences in rMGU were also observed but were artificially reduced by the low blood glucose concentration. To account for this rapid rise of myocardial ${ }^{18} \mathrm{~F}$-FDG in the presence of insulin, early fit was modified to exclude late frame counts (30-60 min) and estimate the linear uptake phase of the curve $(0-10 \mathrm{~min})$. The automated fit of the early frames (from $1.8 \pm 1.5 \mathrm{~min}$ ) generated a marked increase in Patlak $\mathrm{K}_{\mathrm{i}}(P<0.001)$, which also translated to a significantly higher rMGU, compared with controls $(P<0.05)$ (Fig. 5C). Patterns similar to those obtained using the inferior vena cava IDIF were observed when the left ventricular cavity (Supplemental Fig. 3) or left atrial cavity (Supplemental Fig. 4) was used. Optimal-fit restriction combined with the inferior vena cava IDIF dramatically improved the population variability for Patlak $\mathrm{K}_{\mathrm{i}}$ and rMGU, compared with other IDIF volumes (Table 2).

\section{Correlation of IDIF VOIs}

Bland-Altman analysis revealed significant bias in the $\mathrm{K}_{\mathrm{i}}$ calculation using the vena cava IDIF compared with the left atrial cavity IDIF $(-0.084 \pm 0.131 \mathrm{~mL} / \mathrm{g} / \mathrm{min})$, despite a good Pearson correlation. This bias was prominently influenced by the high myocardial counts and spillover to left atrial IDIF in the insulinglucose group. Removal of this group somewhat mitigated the bias $(-0.020 \pm 0.025 \mathrm{~mL} / \mathrm{g} / \mathrm{min})$ and strengthened the Pearson correlation (Fig. 6A). The rMGU was likewise influenced by the insulinglucose group, with a significant shift in the bias when these mice were excluded from Bland-Altman analysis $(-79 \pm 76 \mathrm{vs} .-58 \pm$ $60 \mu \mathrm{mol} / \mathrm{min} / 100 \mathrm{~g}, P<0.01$ ) (Fig. 6B). The Pearson correlation for rMGU was weaker than for Patlak slope, reflecting the added variable of blood glucose levels (Fig. 6B). Correlations to the left ventricular cavity remained significant $(r=0.887, P<0.01)$, though the average difference $(\delta)$ was especially high.

\section{Correlation of Patlak to Semiquantitative Uptake Values}

Optimal-fit Patlak measurements using the vena cava IDIF showed strong correlation to early frame (10-20 min and 20$30 \mathrm{~min}$ ) and weaker correlation to late frame (50-60 $\mathrm{min}$ ) myocardial ${ }^{18}$ F-FDG uptake (\%ID/g) across groups (Fig. 7). Removal of the insulin-glucose group resulted in stronger Pearson correlation of Patlak measures to semiquantitative myocardial uptake over the full acquisition. The correlations were generally stronger for rMGU to \% ID/g (Supplemental Fig. 5).

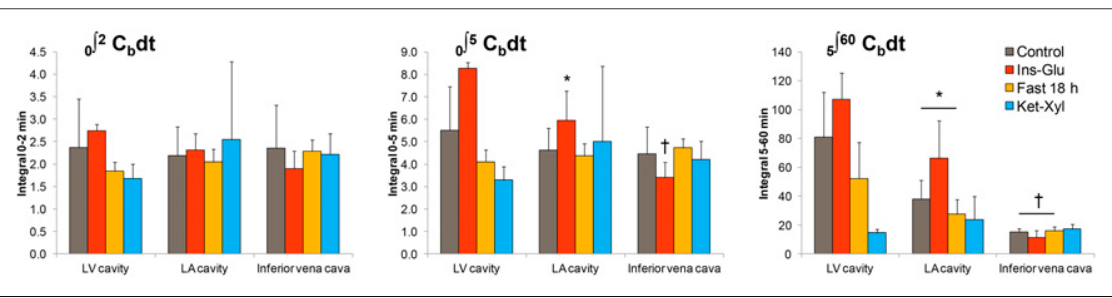

FIGURE 3. Area under the time-activity curve at serial intervals. Integral of blood-pool timeactivity curves over $0-2 \mathrm{~min}, 0-5 \mathrm{~min}$, and 5-60 min of acquisition. ${ }^{*} P<0.01$ to LV cavity, matched group, Student paired $t$ test. ${ }^{\dagger} P<0.01$ to LV cavity and LA cavity, matched group, Student paired $t$ test. Ins-Glu = insulin-glucose; Ket-Xyl = ketamine-xylazine; LA = left atrial; $\mathrm{LV}=$ left ventricular.

\section{DISCUSSION}

The challenge of assigning and using an IDIF for quantitative analysis of glucose metabolism has resulted in a wide range of published glucose utilization values for rodent myocardium. Moreover, a large proportion of preclinical studies makes use of only semiquantitative measurements of myocardial glucose uptake. When assessed with optimal-fit parameters, the Patlak kinetic measurements were consistent with semiquantitative measures of ${ }^{18} \mathrm{~F}-\mathrm{FDG}$ accumulation in myocardium at intermediate 


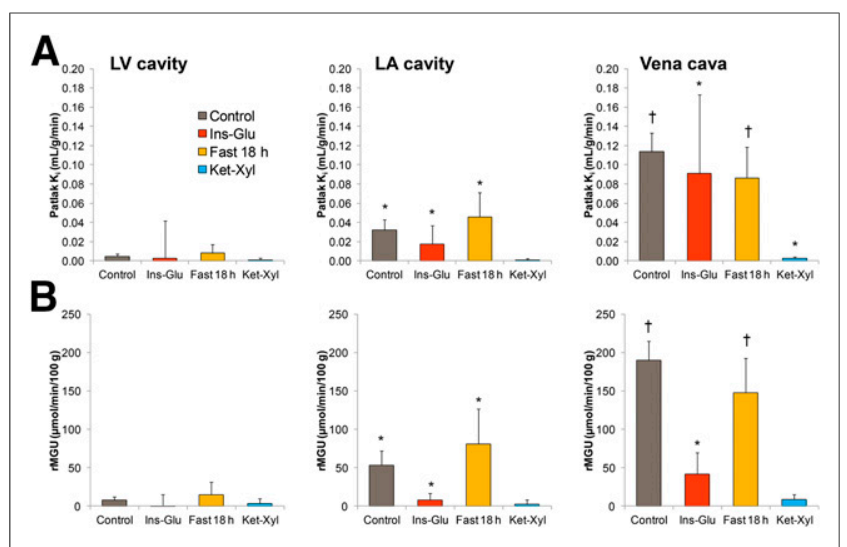

FIGURE 4. Impact of selected blood-pool IDIF on Patlak calculations. Patlak slope $\left(\mathrm{K}_{\mathrm{i}}\right)$ value $(\mathrm{A})$ and $\mathrm{rMGU}(\mathrm{B})$ for each experimental group when using left ventricular cavity, left atrial cavity, or inferior vena cava as blood-pool input function. ${ }^{*} P<0.05$ to LV cavity, matched group, Student paired $t$ test. ${ }^{\dagger} P<0.05$ to LV cavity and LA cavity, matched group, Student paired $t$ test. Ins-Glu = insulin-glucose; Ket-Xyl = ketamine-xylazine; $\mathrm{LA}=$ left atrial; $\mathrm{LV}=$ left ventricular.

and late frames of acquisition, supporting the use of IDIF for repeated imaging studies in small animals. The Patlak calculations using the vena cava IDIF and optimal-fit time intervals compare well with previous reports using a variety of image-derived or blood sampling input functions in control mice $(4,5,11)$, with myocardial Patlak $\mathrm{K}_{\mathrm{i}}$ between 0.08 and $0.15 \mathrm{~mL} / \mathrm{g} / \mathrm{min}$.

Thorn et al. reported on the suitability of the inferior vena cava IDIF for Patlak analysis, applying a recovery coefficient to accurately calculate the activity in the vena cava VOI over time (4). A similar method was used for evaluation of myocardial blood flow using ${ }^{11} \mathrm{C}$-acetate in mice, resulting in reasonable estimation of input function for quantitative imaging (12). The shorter early frames used in the present study ( 2 vs. $10 \mathrm{~s}$ ) may allow sharper definition of the vena cava activity peak and clearance. A third study also evaluated the vena cava as IDIF, demonstrating a modest overestimation $(\sim 10 \%)$ of cerebral Patlak slope, compared with microvolumetric blood counting in rats (13). This discrepancy was somewhat mitigated by application of a dispersion correction, but limitations due to partial-volume errors in the small vena cava of mice remained problematic. In the present study, we calculated consistent IDIF and Patlak measurements in the absence of such corrections with $\mathrm{K}_{\mathrm{i}}$ and rMGU ranges that aligned well with previous reports $(4,13)$.

TABLE 1

Blood Glucose Levels in Experimental Groups

\begin{tabular}{crrrr}
\hline & \multicolumn{4}{c}{ Blood glucose $(\mathrm{mM})$} \\
\cline { 2 - 5 } Group & Prescan & Postscan & Average & Difference \\
\hline Control & $11.1 \pm 2.2$ & $11.7 \pm 2.7$ & $11.4 \pm 1.8$ & $+0.6 \pm 3.3$ \\
Fasting 18h h & $12.4 \pm 1.4$ & $12.6 \pm 4.1$ & $12.5 \pm 2.3$ & $+0.2 \pm 4.0$ \\
$\begin{array}{c}\text { Insulin- } \\
\text { glucose } \\
\text { Ketamine- } \\
\text { xylazine }\end{array}$ & $22.4 \pm 2.8$ & $1.5 \pm 0.5$ & $3.4 \pm 1.3$ & $-3.9 \pm 3.1$ \\
\hline
\end{tabular}

Significant population variability has been reported among mice without metabolic preparation. A reasonable population variability of $17 \%-21 \%\left(\mathrm{~K}_{\mathrm{i}}\right)$ and $13 \%-30 \%$ (rMGU) was obtained among identically treated mice using optimal fits. These values compare favorably with prior values in C57BL/6 (11) and FVB mice (4). By contrast, the left ventricular cavity IDIF resulted in a wider variability of Patlak measurements, reflecting different degrees of late-frame spill-in and curve elevation.

Ideally, the input function for Patlak analysis would be obtained from serial small volumes of arterial blood at regular intervals over the scan. This method has been extensively validated in patients and in rats but is unsuitable for mice because of limited total blood volume. Factor analysis (14), electrocardiogram-gated images (9), and Bayesian decompensation (15) have been proposed as alternate strategies to obtain a reliable IDIF. Although they provide reasonable estimations of the input function in rats, these approaches have not been validated in mice, in which the smaller ventricle size, more rapid first pass, and faster kinetics represent additional challenges. Alternative calculations have used the liver activity (16) or the bladder activity (17) to modify the plasma input function measurements. Hybrid approaches using the combination of IDIF and 1 late $(6,18)$ or up to 6 serial blood samples (5) provided reliable scaling of the IDIF and Patlak measurements. But continued reliance on blood sampling is problematic, particularly in cardiovascular disease models and for repeated scans.

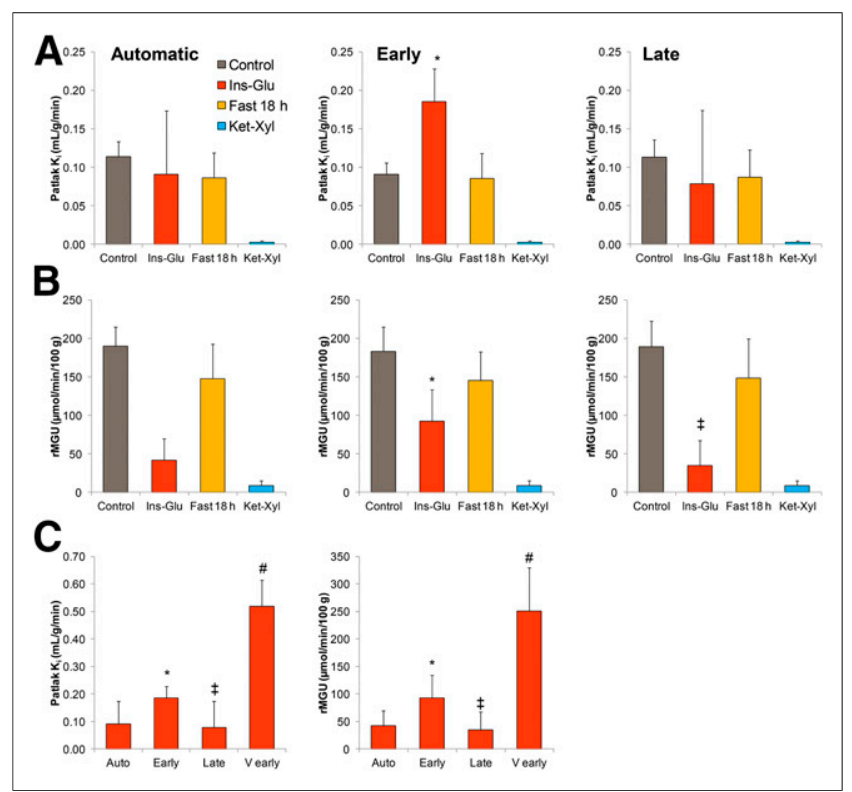

FIGURE 5. Impact of modified temporal limits for kinetic fit on Patlak calculations. Patlak slope $\left(K_{i}\right)$ value $(A)$ and $r M G U(B)$ when automated fit is applied to the full myocardial time-activity curve (automatic), restricting fit from 2 to 40 min (early) or restricting the fit to 10-60 min (late) using inferior vena cava blood pool as input function. (C) Comparison of Patlak $\mathrm{K}_{\mathrm{i}}$ and $\mathrm{rMGU}$ for insulin-glucose group using various time intervals for fitting: automatic ( 7-60 min), early (2-40 min), late (10-60 min), or very early $(0-10 \mathrm{~min})$. ${ }^{*} P<0.05$ to automatic fit, matched group, Student paired $t$ test. $\neq P<0.05$ to early fit, matched group, Student paired $t$ test. $\# P<0.01$ to automatic, early and late fit, matched group, Student paired $t$ test. Auto = automatic; Ins-Glu = insulin-glucose; Ket-Xyl = ketamine-xylazine; $\mathrm{V}$ early = very early. 
TABLE 2

Population Variability with Optimal Fit in Experimental Groups

\begin{tabular}{|c|c|c|c|c|}
\hline \multirow[b]{2}{*}{ Measurement } & \multicolumn{4}{|c|}{ Population variability $\sigma(\%)$} \\
\hline & Control & Fasting $18 \mathrm{~h}$ & Insulin-glucose & Ketamine-xylazine \\
\hline \multicolumn{5}{|l|}{ Patlak $\mathrm{K}_{\mathrm{i}}$} \\
\hline Left-ventricular cavity & 56.6 & 115.9 & 41.4 & 186.9 \\
\hline Left-atrial cavity & 34.3 & 55.1 & 53.4 & 233.5 \\
\hline Inferior vena cava & 17.1 & 21.4 & 18.2 & 73.3 \\
\hline \multicolumn{5}{|l|}{ rMGU } \\
\hline Left-ventricular cavity & 56.6 & 112.8 & 46.9 & 192.8 \\
\hline Left-atrial cavity & 34.7 & 56.2 & 44.8 & 242.2 \\
\hline Inferior vena cava & 13.4 & 30.4 & 31.5 & 69.5 \\
\hline$\sigma=\mathrm{SD} /$ Mean $\times 100 \%$ & & & & \\
\hline
\end{tabular}

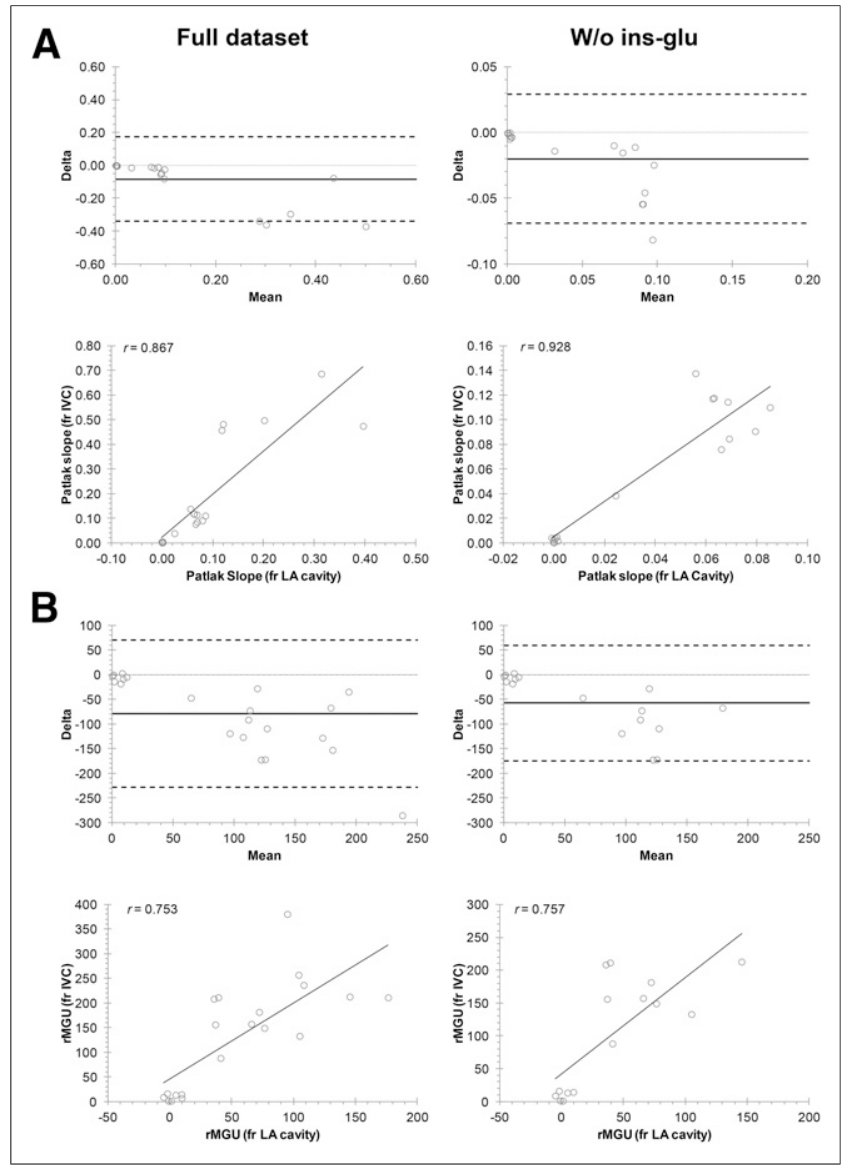

FIGURE 6. Correlation of Patlak values derived using left atrial cavity and vena cava input function. (A) Bland-Altman plot and Pearson correlation comparison of Patlak slope $\left(\mathrm{K}_{\mathrm{i}}\right)$ calculated with left atrial cavity or vena cava-derived input function. Correlation is significantly influenced by high uptake insulin-glucose group. (B) Bland-Altman plot and Pearson correlation comparison of rMGU calculated with left atrial cavity of vena cava-derived input function. Impact of insulin-glucose group on correlation is attenuated for rMGU. fr IVC = from inferior vena cava; $L A=$ left atrial; $w / o$ ins-glu $=$ without insulin-glucose.
The metabolic state of the animal has a marked impact on the shape of the time-activity curve, whereby acute insulin administration precipitates accelerated glucose uptake. This pattern differs considerably from patients, for whom myocardial glucose uptake is relatively continuous. As such, it is essential to consider the initial uptake portion of the curve to derive an accurate indication of glucose utilization in the mouse myocardium. For most animals, the automated fit provided the optimal interpretation of the graphical data. The rapid rise of myocardial ${ }^{18} \mathrm{~F}-\mathrm{FDG}$ after acute insulin treatment, progressing to an apparent plateau of the timeactivity curve, supports more restrictive fit parameters, focusing the Patlak curve to the linear uptake over the first $10 \mathrm{~min}$. The plateau limits the variability from 10 to $60 \mathrm{~min}$, effectively shifting the automated best linear fit to these later points. Acute insulinglucose treatment is useful to reduce the variability of circulating glucose, but the present results suggest deleterious effects on myocardial glucose kinetics. The plateau of the ${ }^{18}$ F-FDG time-activity curve and below-fasting blood glucose levels (i.e., $<5 \mathrm{mM}$ ) suggest rapid glucose depletion, contributing to the lower Patlak slope and rMGU, compared with untreated mice. Moreover, because calculation of rMGU includes blood glucose concentration, this apparent reduction is amplified. Because the maximal effect of insulin on glucose transporter translocation occurs at approximately $30 \mathrm{~min}$, it is reasonable to consider the early portion of the myocardial uptake curve (i.e., $30 \mathrm{~min}$ after intraperitoneal insulin) as representative of insulin-stimulated ${ }^{18} \mathrm{~F}$-FDG uptake. As such, the timing of injection, and balance between insulin and glucose administered, may require more precise titration to obtain favorable kinetics. Furthermore, the plateau of the myocardial ${ }^{18}$ F-FDG timeactivity curve after approximately $20 \mathrm{~min}$ may accurately reflect slowed late-stage glucose uptake kinetics after insulin administration, potentially related to falling glucose concentration, ${ }^{18} \mathrm{~F}-\mathrm{FDG}$ uptake by other insulin-sensitive peripheral organs, or saturation of hexokinase. Nevertheless, using the inferior vena cava as the IDIF and restricting the early Patlak fit to 2-10 min of the acquisition generated reasonable estimations of the Patlak slope and rMGU in acute insulin and glucose-treated mice.

Importantly, the relative difference in $\mathrm{K}_{\mathrm{i}}$ and $\mathrm{rMGU}$ between groups was maintained irrespective of the blood volume selected for the IDIF. As such, although the precise rates of glucose uptake may lack accuracy, comparisons of these values are valid within 

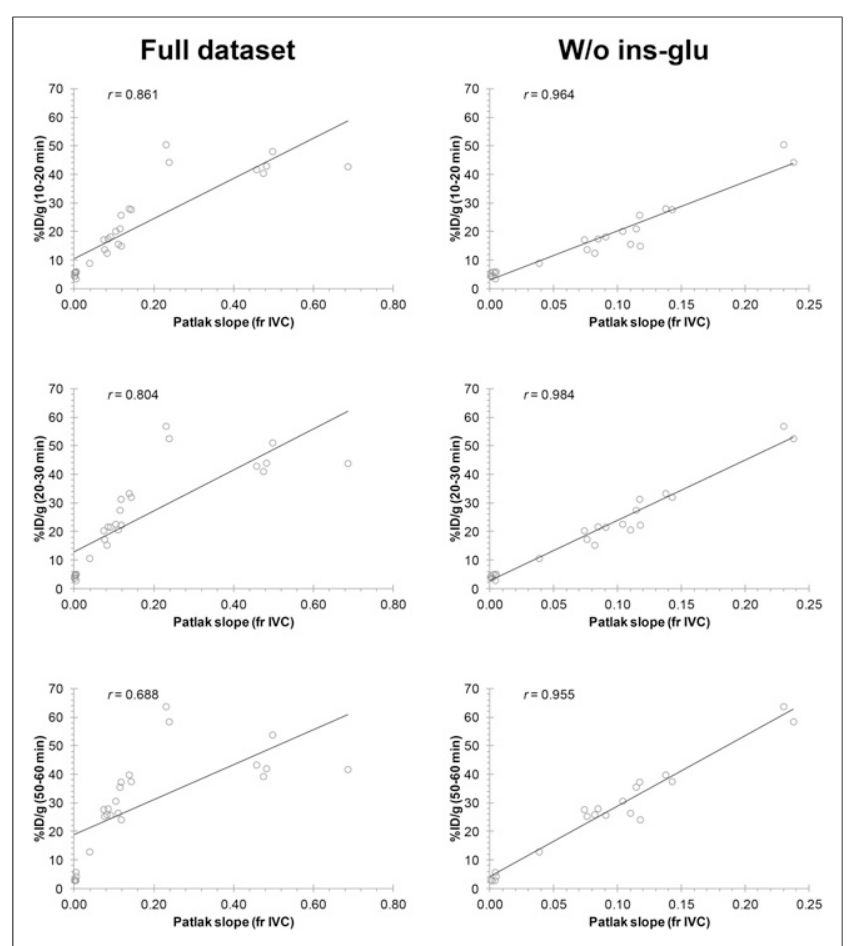

FIGURE 7. Pearson correlation of Patlak values with semiquantitative ${ }^{18} \mathrm{~F}-\mathrm{FDG}$ uptake. Correlation of Patlak slope $\left(\mathrm{K}_{\mathrm{i}}\right)$ to $\% \mathrm{ID} / \mathrm{g}$ in early frames (10-20 min), intermediate frames (20-30 min), and late frames (50$60 \mathrm{~min}$ ) of acquisition. Removal of high and saturated uptake in insulin-glucose group improves correlation. fr IVC = from inferior vena cava; w/o ins-glu $=$ without insulin-glucose.

studies. Moreover, the alignment of optimal Patlak calculations with the mid and late \% ID/g measurements suggests that these semiquantitative estimates provide an accurate representation of the glucose uptake rate.

Several additional issues should be considered when interpreting these results. First, calculation of rMGU depends on the LC to account for the difference in uptake rate and affinity of deoxyglucose versus D-glucose. The constant applied in this study (LC $=0.67$ ) was originally suggested for rabbits and modified for mice and rats (19). As the divisor, the LC proportionally affected the kinetic data, affecting the precision of the rMGU value, but not the relative differences between groups. It is conceivable that the LC is not consistent at all ranges of blood glucose concentrations. Indeed, it has been suggested that at very high concentrations, glucose more strongly competes with ${ }^{18}$ F-FDG uptake (20), which would affect the appropriateness of the LC. Despite this consideration, clear differences in $\mathrm{K}_{\mathrm{i}}$ between groups are apparent, which are independent of the LC, supporting the broad application of the vena cava IDIF and fit parameters.

Second, because it is representative of venous rather than arterial blood, the inferior vena cava blood sample may not be an absolutely accurate surrogate for the input function. In larger mammals, arterial and venous blood concentrations of ${ }^{18} \mathrm{~F}-\mathrm{FDG}$ are thought to reach equilibrium by approximately $30 \mathrm{~min}$. It is, however, reasonable to assume that this equilibrium is attained more rapidly in mice considering the rapid heart rate and small blood volume. Indeed, arterial and venous blood have been frequently considered equivalent as early as 5-10 min after injection (21). Because the hepatic uptake of ${ }^{18} \mathrm{~F}-\mathrm{FDG}$ in mice is very low
( $\sim 3 \%$ ID/g at 50-60 $\mathrm{min}$ ), the contribution of spill-over into the vena cava VOI is considered negligible.

Third, most of the mice evaluated in the present study were under continuous isoflurane anesthesia, which has a pronounced stimulatory effect on myocardial glucose uptake $(22,23)$. Patlak kinetics are certainly augmented by this anesthesia effect, though uptake differences occurring with metabolic preparation or in cardiovascular disease models remain discernible, if somewhat blunted as with fasting (23). Despite several studies purporting the use of alternative anesthetics $(11,24)$, isoflurane remains the most widely used inhalation anesthesia in small-animal imaging.

Fourth, these results are specific to the camera and reconstruction algorithms applied. A performance evaluation of the Inveon camera reported improved definition of the myocardium using the iterative OSEM3D/fastMAP reconstruction as applied here versus filtered backprojection or iterative OSEM2D reconstruction (25). Moreover, the $\beta$ parameter of MAP ( $\beta=1.0$ in the present study) affects the image smoothness, which can influence the clarity and accuracy of the small volumes under evaluation. Cheng et al. demonstrated increased apparent image resolution when the $\beta$ parameter was decreased, at the cost of higher noise (26). It is conceivable that increased noise in the present case would more prominently affect the late frames of the vena cava (raising the apparent activity concentration), and as such a lower $\beta$ parameter may be problematic for calculation. The impact of the $\beta$ parameter and image smoothness on Patlak accuracy remains to be investigated.

And finally, the small dimensions of the vena cava render it highly susceptible to partial-volume effects, which can affect the accuracy of the IDIF. To minimize the difference in potential partial-volume losses between blood-pool VOIs, we maintained a constant transaxial radius $(1 \mathrm{~mm})$. It is reasonable that the partial-volume losses will be greater for smaller structures (i.e., vena cava) than larger structures (i.e., myocardium), which may portend partial-volume correction of the vena cava IDIF for accurate quantitative analysis. Nevertheless, without a correction for partial volume or the application of a recovery coefficient, the uptake values reasonably reflect the kinetic data with a relatively low population variability in identically treated mice.

\section{CONCLUSION}

The inferior vena cava provides a robust and accurate estimation of the blood input function for ${ }^{18} \mathrm{~F}-\mathrm{FDG}$ PET scans in healthy mice, even in the absence of partial-volume or dispersion corrections. The quantification of murine myocardial rate of glucose uptake using an image-derived input function appears to be feasible across a wide range of values, though caution must be exercised in defining the time intervals for fitting Patlak graphical analysis, especially when insulin and glucose are administered at a single time point.

\section{DISCLOSURE}

The costs of publication of this article were defrayed in part by the payment of page charges. Therefore, and solely to indicate this fact, this article is hereby marked "advertisement" in accordance with 18 USC section 1734. This work was supported in part by the REBIRTH-2 Cluster of Excellence and by EU FP7 grant PIRG08GA-2010-276889 (FMB). James T. Thackeray holds a fellowship from the Canadian Institutes of Health Research. No other potential conflict of interest relevant to this article was reported. 


\section{ACKNOWLEDGMENTS}

We thank the preclinical imaging staff for their technical assistance.

\section{REFERENCES}

1. Ménard SL, Ci X, Frisch F, et al. Mechanism of reduced myocardial glucose utilization during acute hypertriglyceridemia in rats. Mol Imaging Biol. 2009; 11:6-14.

2. Shoghi KI, Gropler RJ, Sharp T, et al. Time course of alterations in myocardial glucose utilization in the Zucker diabetic fatty rat with correlation to gene expression of glucose transporters: a small-animal PET investigation. J Nucl Med. 2008;49:1320-1327.

3. Phelps ME, Huang SC, Hoffman EJ, Selin C, Sokoloff L, Kuhl DE. Tomographic measurement of local cerebral glucose metabolic rate in humans with (F-18) 2-fluoro-2-deoxy-D-glucose: validation of method. Ann Neurol. 1979;6: 371-388.

4. Thorn SL, deKemp RA, Dumouchel T, et al. Repeatable noninvasive measurement of mouse myocardial glucose uptake with ${ }^{18} \mathrm{~F}$-FDG: evaluation of tracer kinetics in a type 1 diabetes model. J Nucl Med. 2013;54:1637-1644.

5. Shoghi KI, Welch MJ. Hybrid image and blood sampling input function for quantification of small animal dynamic PET data. Nucl Med Biol. 2007;34: 989-994.

6. Ménard SL, Croteau E, Sarrhini O, et al. Abnormal in vivo myocardial energy substrate uptake in diet-induced type 2 diabetic cardiomyopathy in rats. Am J Physiol Endocrinol Metab. 2010;298:E1049-E1057.

7. Zhong M, Kundu BK. Optimization of a model corrected blood input function from dynamic FDG-PET images of small animal heart. IEEE Trans Nucl Sci. 2013;60:3417-3422.

8. Laforest R, Sharp TL, Engelbach JA, et al. Measurement of input functions in rodents: challenges and solutions. Nucl Med Biol. 2005;32:679-685.

9. Locke LW, Berr SS, Kundu BK. Image-derived input function from cardiac gated maximum a posteriori reconstructed PET images in mice. Mol Imaging Biol. 2011;13:342-347.

10. Thackeray JT, Bankstahl JP, Wang Y, et al. Targeting post-infarct inflammation by PET imaging: comparison of ${ }^{68} \mathrm{Ga}$-citrate and ${ }^{68} \mathrm{Ga}$-DOTATATE with ${ }^{18}$ F-FDG in a mouse model. Eur J Nucl Med Mol Imaging. 2015;42:317327.

11. Kreissl MC, Stout DB, Wong KP, et al. Influence of dietary state and insulin on myocardial, skeletal muscle and brain $[\mathrm{F}]$-fluorodeoxyglucose kinetics in mice. EJNMMI Res. 2011;1:8.
12. Croteau E, Renaud JM, Archer C, et al. $\beta 2$-adrenergic stress evaluation of coronary endothelial-dependent vasodilator function in mice using $\mathrm{C}$-acetate micro-PET imaging of myocardial blood flow and oxidative metabolism. EJNMMI Res. 2014;4:68.

13. Lanz B, Poitry-Yamate C, Gruetter R. Image-derived input function from the vena cava for ${ }^{18}$ F-FDG PET studies in rats and mice. J Nucl Med. 2014;55:13801388 .

14. Kim J, Herrero P, Sharp T, et al. Minimally invasive method of determining blood input function from PET images in rodents. J Nucl Med. 2006;47:330-336.

15. Mabrouk R, Dubeau F, Bentourkia M, Bentabet L. Extraction of time activity curves from gated FDG-PET images for small animals' heart studies. Comput Med Imaging Graph. 2012;36:484-491.

16. Green LA, Gambhir SS, Srinivasan A, et al. Noninvasive methods for quantitating blood time-activity curves from mouse PET images obtained with fluorine18-fluorodeoxyglucose. J Nucl Med. 1998;39:729-734.

17. Wong KP, Zhang X, Huang SC. Improved derivation of input function in dynamic mouse $\left[{ }^{18} \mathrm{~F}\right] \mathrm{FDG}$ PET using bladder radioactivity kinetics. Mol Imaging Biol. 2013;15:486-496.

18. Schiffer WK, Mirrione MM, Dewey SL. Optimizing experimental protocols for quantitative behavioral imaging with ${ }^{18}$ F-FDG in rodents. J Nucl Med. 2007; 48:277-287.

19. Krivokapich J, Huang SC, Selin CE, Phelps ME. Fluorodeoxyglucose rate constants, lumped constant, and glucose metabolic rate in rabbit heart. Am J Physiol. 1987;252:H777-H787.

20. Ng CK, Holden JE, Degrado TR, Raffel DM, Kornguth ML, Gatley SJ. Sensitivity of myocardial fluorodeoxyglucose lumped constant to glucose and insulin. Am J Physiol. 1991;260:H593-H603.

21. Shimoji K, Ravasi L, Schmidt K, et al. Measurement of cerebral glucose metabolic rates in the anesthetized rat by dynamic scanning with ${ }^{18} \mathrm{~F}-\mathrm{FDG}$, the ATLAS small animal PET scanner, and arterial blood sampling. J Nucl Med. 2004;45:665-672.

22. Thackeray JT, Bankstahl JP, Wang Y, Wollert KC, Bengel FM. Clinically relevant strategies for lowering cardiomyocyte glucose uptake for ${ }^{18} \mathrm{~F}-\mathrm{FDG}$ imaging of myocardial inflammation in mice. Eur J Nucl Med Mol Imaging. 2015;42:771-780.

23. Fueger BJ, Czernin J, Hildebrandt I, et al. Impact of animal handling on the results of ${ }^{18}$ F-FDG PET studies in mice. $J$ Nucl Med. 2006;47:999-1006.

24. Toyama H, Ichise M, Liow JS, et al. Evaluation of anesthesia effects on $\left[{ }^{18} \mathrm{~F}\right]$ FDG uptake in mouse brain and heart using small animal PET. Nucl Med Biol. 2004;31:251-256.

25. Constantinescu CC, Mukherjee J. Performance evaluation of an Inveon PET preclinical scanner. Phys Med Biol. 2009;54:2885-2899.

26. Cheng JC, Shoghi K, Laforest R. Quantitative accuracy of MAP reconstruction for dynamic PET imaging in small animals. Med Phys. 2012;39:1029-1041. 